\title{
A importância da assistência aos pacientes em cuidados paliativos na atenção primária
}

\author{
The importance of patient assistance in palliative care in primary care
}

La importancia de la asistencia al paciente en cuidados paliativos en atención primaria

Mayra Loures de Oliveira ${ }^{\text {* }}$, Mônica Isaura Corrêa ${ }^{2}$, Amanda Garcia da Costa ${ }^{1}$, Ana Karoline Santos Losada $^{3}$, Anna Cláudia de Campos Venâncio ${ }^{1}$, Daniela Cavalcante Gomes ${ }^{4}$, Gabrielle de Oliveira Pires $^{5}$, Larisse Ribeiro Bastos ${ }^{5}$, Matheus Rodrigues da Silva ${ }^{1}$, Victória Marques de Figueiredo Sabino ${ }^{1}$.

\section{RESUMO}

Objetivo: Esse artigo buscou discutir a importância dos cuidados paliativos na assistência aos pacientes, trazendo o foco para a atenção básica como fator crucial para a integralidade do cuidado. Revisão bibliográfica: $O$ trabalho avalia o papel da equipe da atenção básica na contribuição para o aprimoramento e para a difusão da assistência aos pacientes com doenças incuráveis, destacando a necessidade de serem realizados desde o nível primário de atenção em saúde, com o objetivo de oferecer aos pacientes melhor qualidade de vida e atenuação do sofrimento logo no início do processo de diagnóstico, para que todo o processo saúde-doença possa ser considerado mais digno. Considerações finais: Nesse sentido, o artigo também traz a reflexão sobre a realidade dos cuidados paliativos no sistema nacional de saúde, evidenciando a carência de uma legislação e a necessidade da criação de parâmetros oficiais e estratégias que assegurem os direitos dos pacientes conforme os princípios de integralidade, universalidade e equidade que regem nosso Sistema Único de Saúde (SUS).

Palavras-chave: Cuidados paliativos, Atenção básica, Saúde.

\begin{abstract}
Objective: This article sought to discuss the importance of palliative care in patient care, focusing on primary care as a crucial factor for comprehensive care. Literature review: The work assesses the role of the primary care team in contributing to the improvement and dissemination of assistance to patients with incurable diseases, highlighting the need to be carried out from the primary level of health care, with the aim of offering patients better quality of life and mitigation of suffering early in the diagnosis process, so that the entire healthdisease process can be considered more dignified. Final considerations: In this sense, the article also reflects on the reality of palliative care in the national health system, highlighting the lack of legislation and the need to create official parameters and strategies that ensure the rights of patients according to the principles of comprehensiveness, universality and equity that govern our Unified Health System (SUS).
\end{abstract}

Key words: Palliative care, Basic care, Health.

\section{RESUMEN}

Objetivo: Este artículo buscó discutir la importancia de los cuidados paliativos en la atención al paciente, enfocándose en la atención primaria como factor crucial para la atención integral. Revisión bibliográfica: El trabajo evalúa el papel del equipo de atención primaria en la contribución a la mejora y difusión de la atención a los pacientes con enfermedades incurables, destacando la necesidad de realizarse desde el nivel primario de atención, con el objetivo de ofrecer una mejor atención a los pacientes. calidad de vida y mitigación del sufrimiento temprano en el proceso de diagnóstico, para que todo el proceso salud-enfermedad pueda

${ }^{1}$ Centro Universitário de Caratinga (UNEC), Caratinga - MG. *E-mail: mayralouresol@gmail.com

2 Faculdade de Medicina do Vale do Aço (FAMEVAÇO), Ipatinga - MG.

${ }^{3}$ Faculdades Integradas do Norte de Minas (FUNORTE), Montes Claros - MG.

${ }^{4}$ Centro Universitário Uninovafapi (UNVFP), Teresina - PI.

${ }^{5}$ Centro Universitário de Volta Redonda (UNIFOA), Volta Redonda - RJ.

SUBMETIDO EM: 2/2021

ACEITO EM: 3/2021

PUBLICADO EM: $3 / 2021$ 
considerarse más digno. Consideraciones finales: En este sentido, el artículo también trae una reflexión sobre la realidad de los cuidados paliativos en el sistema nacional de salud, destacando la falta de legislación y la necesidad de crear parámetros y estrategias oficiales que aseguren los derechos de los pacientes de acuerdo con los principios de integralidad, universalidad y equidad que gobiernan nuestro Sistema Único de Salud (SUS).

Palabras clave: Cuidados paliativos, Cuidado básico, Salud.

\section{INTRODUÇÃO}

Cuidados Paliativos (PC) é definido como um atendimento integral ativo oferecido à pessoas que estão enfrentando um momento angustiante relacionado à sua saúde, resultante de doenças graves que afetam a qualidade de vida. A abordagem visa, principalmente, a prevenção e o alívio do sofrimento, com o objetivo de confortar o paciente, seus familiares e cuidadores a fim de proporcioná-los bem-estar e, redução dos sintomas e aflições que o enfrentamento de enfermidades pode gerar (JUSTINO ET, et al., 2020).

A Atenção Primária de Saúde (APS) é um fator de extrema importância para o desenvolvimento dos cuidados paliativos, já que é crucial nas políticas de saúde e amplia o acesso à população. No sistema de saúde brasileiro, o protótipo de APS é centrado no Programa de Saúde da Família (PSF), distribuído nacionalmente, com a proposta de um atendimento integral do paciente em sua dimensão individual, familiar e social (JUSTINO ET, et al., 2020).

Concomitante à crescente expectativa de vida, o número de ocorrência de mortalidade da população por doenças crônicas degenerativas não transmissíveis apresentou um significativo aumento, tornando a demanda por cuidados paliativos um problema de saúde pública. Evidencia-se a relevância do PSF nesse processo, uma vez que já propõe visitas domiciliares por profissionais de saúde e o cuidado integral. Entretanto, a implementação dos cuidados paliativos não é apenas técnica com equipes multidisciplinares, já que envolve questões éticas no relacionamento entre profissionais, família e o paciente, e a carência de políticas que articulem estratégias para os cuidados de controle dos sintomas desde o diagnóstico até o luto. A assistência domiciliar tem um papel preponderante já que permite a humanização dos cuidados que compreendem ações de suporte do cotidiano como higiene, alimentação etc. como também um amparo afetivo aos pacientes (QUEIROZ AHAB, et al., 2013).

Nesse contexto, é necessário destacar a dificuldade de identificação de pacientes que necessitam desses tipos de cuidados e suas condições sociais. Além disso, é notório o despreparo profissional em lidar com situações que envolvem o óbito, principalmente relacionadas à comunicação à família, que, muitas das vezes, negligencia as fases do processo morte por estar sobrecarregada pelos cuidados ao doente (SOUZA HI, et al., 2015; JUSTINO ET, et al., 2020).

Assim, o objetivo deste trabalho é discutir a assistência aos pacientes em cuidados paliativos na atenção básica, evidenciando sua importância. Além de abordar o desafio que envolve a implementação regulamentada por lei de acordo com as políticas sociais que garantem o acesso à saúde como direito básico, e o preparo profissional desde a faculdade.

\section{REVISÃO BIBLIOGRÁFICA}

Os denominados cuidados paliativos são atribuídos aos pacientes com doenças crônicas evolutivas que oferecem riscos a suas vidas. Têm como foco principal manter a qualidade de vida, do momento de descoberta da doença à sua fase final, e sempre alinhado com a doutrina do Sistema Único de Saúde (SUS): equidade, universalidade e integralidade (BRASIL, 2015).

Portanto, esse tipo de cuidado está inserido em todos os cuidados da área da saúde e respeita o conceito de níveis de atenção no âmbito do SUS e, na maioria das vezes, é um cuidado aprofundado para o conforto e gestão do ciclo de vida. No entanto, o exame deve ser iniciado imediatamente após o diagnóstico e reforçado quando necessário. Incluem o direito das pessoas a receberem apoio e assistência ao longo da vida da 
doença até o fim da vida. Os cuidados paliativos podem afirmar a vida e tratar a morte como um processo normal, e não significa acelerar ou retardar a morte. Nesse caso, eles integram os aspectos psicossociais e espirituais ao cuidado do paciente, fornecem um sistema de apoio e os ajudam a viver o mais ativamente possível até a morte. Além disso, fornece um sistema de apoio para ajudar os pacientes, juntamente com seus familiares, a lidar com situações durante a doença e o luto, que necessitam de trabalho em equipe e atenção contínua até a manutenção dos resultados da dinâmica familiar. Por fim, ressaltaram que desde o estágio inicial da doença, por meio de modificações e tratamentos que prolongam a vida, são aplicáveis até o final do processo de luto (WORLD HEALTH ORGANIZATION, 2002).

Dessa maneira, a assistência paliativa não deve ser vista como uma opção após um tratamento ineficaz da doença, mas uma teia de cuidados voltados ao paciente e um tipo específico de atendimento, que é organizado para ser concedido a população em todos os níveis de referência, atentando-se ao princípio de integralidade do Sistema Único de Saúde (SUS). Dessa forma, pelo fato do acompanhamento acontecer do momento do descobrimento da doença até a morte do paciente, a abordagem se torna mais específica para que o indivíduo viva da melhor maneira possível, além de uma relação médico-paciente mais íntima e de confiança (BOEMER M, et al., 2009).

Sendo assim, enfatiza-se a importância de evidenciar que "cuidados paliativos" não é o mesmo que Atendimento Domiciliar do tipo "internação domiciliar", mas que este é um recurso de assistência muito utilizado. Sendo assim, os cuidados paliativos procuram, por meio de um conjunto de medidas, por fim aos sintomas mais severos que afligem o paciente com uma enfermidade já em fase avançada para, a partir de uma melhora, tentar garantir qualidade a sua rotina, ou diminuir o sofrimento, e ofertar as medidas cabíveis para que o decorrer do atendimento seja considerado mais digno, entrando em acordo com o paciente e familiares a respeito de quais passos serão seguidos (MARCELINO SR, et al., 2000).

Nesse viés, refletir sobre o papel das equipes de atenção básica nos cuidados paliativos pode ajudar a refinar e difundir a importância dessa disciplina tão empática. Compreensivelmente, essa combinação pode ajudar a reduzir o descaso e o sofrimento dos pacientes e familiares. Portanto, além da importância da inclusão dos cuidados paliativos no sistema nacional de saúde, e da importância de cada vez mais integrar a rede de atenção básica com a rede hospitalar, também é necessário considerar a organização de alguns pontos de forma adequada para participar da implantação dos cuidados paliativos; à interação das relações familiares que, muitas vezes, são afetadas no inevitável processo evolutivo da doença; organizar recursos humanos qualificados para composição da equipe, ou seja, profissionais especializados e com preparo emocional para lidar com pacientes terminais e suas famílias (QUEIROZ AHAB, et al., 2013).

Por volta dos anos 2000 , ocorreu um aumento significativo nos cuidados paliativos, porém ainda pautado em uma desigualdade no Brasil (ACADEMIA NACIONAL DE CUIDADOS PALIATIVOS (ANCP), 2013). Isso acontece devido há uma concentração notória em São Paulo, revelando dessa maneira como as regiões do Brasil possuem diferenças, não só nesse quesito, mas na oferta da maioria dos serviços públicos. De acordo com dados publicados no Atlas de Cuidados Paliativos en Latinoamerica, ao contrário do que tem acontecido em vários países do mundo, o Brasil ainda carece de legislação sobre cuidados paliativos. Além disso, não existem parâmetros oficiais pré-estabelecidos para mensurar a verdadeira necessidade de atuação nessa área, uma vez que atualmente é utilizada a expectativa de vida de pelo menos seis meses (ASOCIACIÓN LATINOAMERICANA DE CUIDADOS PALIATIVOS, 2013; ANCP, 2012).

Dessa maneira, para que as organizações que prestam cuidados paliativos atinjam os objetivos que são impostos, é necessário uma gestão na estrutura organizacional. Assim, para transformar a realidade brasileira em relação à qualidade dos processos que atingem diretamente a população, é esperado uma mudança de gestão. No caso do setor público, é preciso que as ações sejam desenvolvidas e realizadas de maneira eficiente, prática e transparente, para que dessa forma os seus resultados sejam favoráveis a mudança da realidade do país e, que assim gere uma certa esperança por parte da sua população (FROSSARD AN, 2016). Visto que, um dos entraves para viabilizar CP, é que os tratamentos curativos possuem prioridade no quesito verba destinada, sendo necessário reforçar a importância dos $\mathrm{CP}$, para os gestores, já que, geralmente, tratase da única opção para os pacientes que possuem doenças crônico-degenerativas (BOEMER M, et al., 2009). 
O ensino das faculdades de medicina encontra-se baseado no modelo biomédico, onde é ensinado ao futuro praticante que o fim último de qualquer terapêutica seria a cura de uma doença, provocando o falso ideal de que todas as doenças seriam passíveis de cura. Além deste fato, com a era moderna nota-se o crescimento de métodos diagnósticos e terapêuticos que prolongam a vida do paciente, levando novamente a falsa idéia da onipotência do médico frente à doença (FIGUEIREDO MTA, 2003).

O estudo atento da semiótica do modelo biomédico leva a compreensão de que o profissional atual é extremamente competente para lutar contra a doença, lançando mão a tratamentos e métodos diagnósticos para interromper a progressão desta, porém, nesta batalha travada entre a patologia e o médico, muita vezes perde-se de vista o corpo onde essa doença habita, ou seja, o paciente (FIGUEIREDO MGMCA e STANO RCMT, 2013).

Neste cenário, com o alto índice de doenças que ameaçam a vida, faz-se necessário a mudança do modelo biomédico para o biopsicossocial, nas faculdades de medicina. Visto que o indivíduo e o adoecer passarão a serem vistos de maneira integral, englobando as dimensões físicas, sociais e psicológicas, o que permite ao estudante de medicina o aprendizado de capacidades relacionais, as quais são fundamentais para o CP (MARCOS MA, 2006).

A especialidade dos cuidados paliativos têm como princípio o conforto de um paciente que se encontra em uma situação em que não há uma possível cura, tendo sua origem logo nos primórdios da medicina. Já na época de Hipócrates, ele refere como o dever do médico "curar quando possível, aliviar quando a cura não for possível e consolar quando não houver mais nada a fazer". Os Cuidados Paliativos não se apresentam como uma forma de negação da prática biomédica, uma vez que são extremamente intervencionistas na tentativa de diminuir o sofrimento do paciente, mas de um lembrete da finitude humana e da lembrança da necessidade de olhar para o ser presente com a doença de forma empática e multifacetada (BURLÁ C e PY L, 2014). Etimologicamente a palavra paliativa deriva do termo em latim pallium, que significa manto, ou seja, seria a prática de uma medicina que tem como seu fim último não a cura de uma doença, mas a diminuição do sofrimento e sintomas desagradáveis e incapacitantes de um paciente (FONSECA A e GEOVANINI F, 2013).

O trabalho em cuidados paliativos se encontra em uma práxis multiinterprofissional, onde se encontram médicos, enfermeiros, técnicos em enfermagem, fonoaudiólogos, psicólogos, nutricionistas e assistentes espirituais, devendo ter a mesma valorização e trabalharem em conjunto com o intuito de tratar o paciente em uma óptica biopsicossocial, ou seja, olhando para este de forma holística e não focada exclusivamente na doença do paciente (KASTOR KS, et al., 2019; GOMEZ ALZ e OTHERO MB, 2016).

O profissional médico paliativista deve possuir as competências de comunicação verbal e não-verbal, trabalho em equipe, compreensão da terminalidade e do luto, empatia perante o paciente e sua família, além do saber profundo da ciência médica, sendo que este deve utilizar métodos para garantir o bem-estar de seu paciente, manejando drogas como analgésicos, sedativos, reguladores intestinais, antidepressivos e antipsicóticos (FONSECA A, GEOVANINI F, 2013). Além disso, é necessário que o conhecimento acerca dos $\mathrm{CP}$, seja expandido aos médicos generalistas, visando o baixo número de médicos especialistas na área e 0 alto índice de demanda (SOUZA HL, et al., 2015).

Estas habilidades se fazem primordiais e fundamentais, visto que os profissionais da saúde presentes na atenção primária precisam lidar com questões técnicas, mas sobretudo éticas, tais como: parecer sobre qual será o local de morte do paciente, forma como irão lidar com o luto e a vivência de assistir à morte, identificação e manejo das condições que o paciente e os familiares apresentam para a abordagem dos cuidados paliativos em seu domicílio, que geram a necessidade de utilizar tais habilidades, para que 0 pacientes recebam apoio e acolhimento necessário. Além de criar um vínculo sólido com a família para que as informações sejam passadas de forma franca e honesta bilateralmente, a fim de estabelecer estratégias mais eficazes (SOUZA HL, et al., 2015).

O surgimento dos Cuidados Paliativos no Brasil data de 1980, período do fim ditatorial, iniciando-se nos estados de São Paulo, Rio Grande do Sul e Santa Catarina, tendo sido aprovada como especialidade médica 
em 2011 pelo Conselho Federal de Medicina (CFM), porém, sua prática ainda se encontra restrita a poucos locais (FONSECA A e GEOVANINI F, 2013). Segundo a ANCP com dados coletados no ano de 2018, no Brasil havia 177 equipes de Cuidados Paliativos, dos mais de 5 mil hospitais, observa-se que apenas $10 \%$ apresentavam estas equipes e que 50\% delas concentram-se na região Sudeste (ANCP, 2018).

O envelhecimento da população mundial traz consigo o aumento de doenças crônicas, sem ser acompanhado de melhoria na qualidade de vida e no enfrentamento das enfermidades. Nesse contexto, os cuidados paliativos se enquadram como um método interdisciplinar, onde cada profissional reconhece seu limite de atuação para contribuir com o paciente em estado terminal, o que visa garantir o cuidado integral com o ser humano (ORTHI L, et al., 2019).

Dessa forma, os cuidados paliativos possuem alguns princípios que incluem a importância da vida, considerando a morte como um processo natural, o estabelecimento de um cuidado que não acelere a chegada da morte e nem a prolongue com medidas desproporcionais (obstinação terapêutica), a propiciação do alívio da dor e de outros sintomas penosos; integrar os aspectos psicológicos e espirituais na estratégia do cuidado e oferecer um sistema de apoio a família para que ela possa enfrentar a doença do paciente e sobreviver ao período de luto (HERMES H e LAMARCA I, 2013).

Diante disso, a assistência de pacientes em cuidados paliativos se dá por meio de visitas domiciliares feitas por médicos, enfermeiros, psicólogos, fisioterapeutas e outros profissionais de saúde que visam melhorar a qualidade de vida remanescente, incluindo a redução da probabilidade de visitas hospitalares perto do fim da vida (TANUSEPUTRO $P$, et al., 2018). Para que esse trabalho seja realizado de maneira efetiva conta-se com algumas estratégias, sendo a primeira delas a escuta ativa, que tenta desenvolver no paciente a sensação de "ser compreendido"; em seguida adota-se a aceitação com o outro, o que permite desenvolver uma aceitação compreensiva e não normativa; além da empatia, a qual é o esforço para compreender os sentimentos das outras pessoas; e a confiança que é construída no desenrolar da conversa com o paciente e seus familiares que se desenvolve a partir de uma tomada de consciência das próprias emoções do profissional e no manejo adequado na direção da ação terapêutica (LOPES M e SILVA J, 2004).

\section{CONSIDERAÇÕES FINAIS}

Com base na bibliografia deste estudo, o presente artigo traz a importância dos cuidados paliativos como ferramenta para melhoria da qualidade de vida e atenuante do sofrimento do paciente com uma doença dita "incurável". Esse trabalho, realizado pela atenção primária, é considerado um direito do ser humano, e deve ser assegurado, seguindo as doutrinas do Sistema Universal de Saúde (SUS). Para que seja realizado de forma satisfatória, deve-se, portanto, envolver o lado empático do médico, uma organização sistêmica, em que envolva legislação adequada, articulação entre a rede básica e a rede hospitalar, envolvimento de equipe multidisciplinar que esteja capacitada tecnicamente e possua competências para acolher esses pacientes, apoio e assistência a família do paciente.

\section{REFERÊNCIAS}

1. ASOCIACIÓN LATINO-AMERICANA DE CUIDADOS PALIATIVOS, 2013. IN: Dicionário espanhol. Disponível em: https://cuidadospaliativos.org. Acessado em: 22 de set. 2020.

2. ANCP - ACADEMIA NACIONAL DE CUIDADOS PALIATIVOS. Manual de Cuidados Paliativos, 2012.

3. ANCP - ACADEMIA NACIONAL DE CUIDADOS PALIATIVOS. Manual de Cuidados Paliativos, 2013.

4. ANCP - ACADEMIA NACIONAL DE CUIDADOS PALIATIVOS. Divulga Panorama dos Cuidados Paliativos no Brasil. 2018. In: ANCP. Brasil. Disponível em: https://paliativo.org.br/ancp-divulga-panorama-dos-cuidados-paliativos-nobrasil/. Acessado em: 13 set. 2020.

5. BRASIL. Ministério da Saúde. Política Nacional de Atenção Oncológica: Desde a Promoção até os últimos cuidados, dados esses como Paliativos, 2005.

6. FIGUEIREDO MTA. Educação em cuidados paliativos: uma perspectiva brasileira. Mundo Saúde, 2003; 27(1): 165170.

7. FIGUEIREDO MGMCA, STANO RCMT. O estudo da morte e dos cuidados paliativos: uma experiência didática no currículo de medicina. Revista Brasileira de Educação Médica, 2013; 37 (2): 298-306. 
8. FONSECA A, GEOVANINI F. Cuidados paliativos na formação do profissional da área da saúde. Revista Brasileira de Educação Médica, 2013; 37 (2): 120-125.

9. FROSSARD A. Os cuidados paliativos como política pública: notas introdutórias. Cadernos EBAPE, 2016; 4: 640-655

10. GOMES ALZ, OTHERO MB. Cuidados paliativos. Estudos Avançados, 2016; 30 (88): 155-166.

11. HERMES H, LAMARCA I. Cuidados paliativos: uma abordagem a partir das categorias profissionais de saúde. Ciência e saúde coletiva, 2013; 18(9): 2577-2588

12. JUSTINO ET, et al. Cuidados paliativos na atenção primária à saúde: revisão do escopo. Rev. Latino-Am. Enfermagem. 2020; 28:3324.

13. KASTOR KS, et al. Cuidados paliativos: Perfil com olhar biopsicossocial dentre pacientes oncológicos. Sociedade Brasileira de Estudos de Dor, 2019; 2 (1): 49-54.

14. LOPES M, SILVA J. Estratégias metodológicas de educação e assistência na atenção básica de saúde. Revista latino-am Enfermagem, 2004; 12(4): 683-688.

15. MARCELINO SR, et al. Cuidado domiciliar: escolha ou falta de opção? Texto \& Contexto Enfermagem 2000; 9:9-21.

16. MARCUCCI FCl, et al. Identificação de pacientes com indicação de Cuidados Paliativos na Estratégia de Saúde da Família: estudo exploratório. Cad. saúde coletiva, 2016; 24(2): 145-152.

17. ORTHI L, et al. Conhecimento do acadêmico de Medicina sobre Cuidados paliativos. Revista Brasileira de Educação Médica, 2019; 43: 286-295.

18. QUEIROZ AHAB, et al. Percepção de familiares e profissionais de saúde sobre os cuidados no final da vida no âmbito da atenção primária à saúde, Ciênc. saúde coletiva, 2013; 18(9): 2615-2623.

19. TANUSEPUTRO $P$, et al. Associações entre visitas domiciliares de médicos para o moribundo e o local da morte: $A$ estudo de coorte retrospectivo de base populacional. Plosone, 2018; 13 (2): 1-14.

20. WORLD HEALTH ORGANIZATION (WHO). National cancer control programmes: policies and managerial guidelines. 2. ed. Genebra: OMS, 2002.

21. BURLÁ C, PY L. Cuidados paliativos: ciência e proteção ao fim da vida. Caderno de Saúde Pública, $2014 ; 30$ (6): $1139-1141$

22. BOEMER M. Sobre cuidados paliativos. Rev. esc. enferm. USP, 2009; 43(3).

23. SOUZA HL, et al. Cuidados paliativos na atenção primária à saúde: considerações éticas. Rev. Bioét. [online], 2015; 23(2): 349-359.

24. MARCO MA. Do modelo biométrico ao modelo biopsicossocial: um projeto se educação permanente. Rev. bras. educ. med., 2006; 30. 